\title{
ALFVÉN WAVES AND RADIATION PRESSURE IN THE WINDS OF WOLF-RAYET STARS
}

\author{
L. C. DOS SANTOS, V. JATENCO-PEREIRA and R. OPHER \\ Instituto Astronômico e Geofísico - Universidade de São Paulo, Caixa Postal 9638 CEP \\ 01065-970 - São Paulo - SP - Brazil
}

\begin{abstract}
We recently showed that Alfvén waves acting jointly with radiation pressure in Wolf-Rayet winds can possibly solve the momentum problem (dos Santos, Jatenco-Pereira and Opher 1993a, 1993b). In these analyses, however, constant radiation luminosity as a function of the distance to the star was assumed. We impose here conservation of the energy flux and examine the effect on the radiation luminosity.
\end{abstract}

Key words: stars: Wolf-Rayet - winds - Alfvén waves

\section{Introduction}

The existence of stellar winds around early-type stars is now fairly well established. As demonstrated first by Lucy \& Solomon (1970), the radiative momentum absorbed by UV spectral lines is able to initiate stellar winds, since the radiative line acceleration exceeds the gravity by a large factor. The first model to derive mass-loss rates, $\dot{M}$, and flow speeds in good agreement with observations was that of Castor, Abbot \& Klein (1975) (CAK). One of the major difficulties presented by the radiation driven wind theory is the momentum problem in Wolf-Rayet stars, which can be described using the ratio $\eta=\left(\dot{M} v_{\infty}\right) /\left(L_{*} / c\right)$, where $v_{\infty}$ the terminal velocity and $L_{*}$ is the luminosity of the star. Barlow et al. (1981) found that for WR stars $\eta$ ranges from 4 to 53 . To get around the momentum problem one cannot simply appeal to a larger luminosity, because the values that are used cannot be near the Eddington limit (Cassinelli \& van der Hucht 1987).

Recently dos Santos, Jatenco-Pereira \& Opher (1993a, 1993b) developed a model for mass-loss in WR stars, where both a flux of Alfvén waves and radiation pressure are important. This work indicates that Alfvén waves acting jointly with radiation pressure, provide energy and momentum for the wind with reasonable Alfvén wave fluxes and magnetic fields.

\section{The Model}

The model studied is a fusion of the Alfvén wave wind model of JatencoPereira \& Opher (1989a, 1989b, 1989c) (JPO) and the radiation pressure model of CAK. We assume that a magnetic field exists in WR stars, and that the geometry of this field is similar to that of a solar coronal hole.

We solve the equation of motion (i.e., Eq. (52) of Holzer, Fla \& Leer 1983), and use an effective escape velocity which takes into account the 
CAK power index expressing the effect of all lines, possible nonsolar abundances, and the finite size of the disk of the star (see Kudritzki \& Hummer $1990)$. We use a flux of Alfvén waves $\left(\phi_{M_{o}}\right)$ generated near the stellar surface, $R_{*}$, (possibly by the annihilation of twisted magnetic fields, e.g., Opher $\&$ Pereira 1986) as a mechanism (jointly with pressure radiation) of heating and accelerating the stellar wind, with a damping length, $L$. We study a damping process investigated previously by JPO, damping due to turbulence. We impose conservation of energy flux, and examine the effect of the transformation of the radiation energy into the energy of the wind.

\section{Results}

The parameters of the standard WR stars studied are: $R_{*}=12 \mathrm{R}_{\odot}, M_{*}=16$ $\mathrm{M}_{\odot}, L_{*}=10^{5.1} \mathrm{~L}_{\odot}, \Gamma_{\text {Edd }}=0.214$ and $\dot{M}=10^{-4.7} \mathrm{M}_{\odot} \mathrm{yr}^{-1}$. We use a spherical symmetry for the open magnetic field lines and the initial damping length $L \sim 10.0 R_{*}$.

The preliminary result of our investigation of the ratio of wind velocity to escape velocity as a function of the distance from the star, for $\phi_{M_{0}}=$ $6.0 \times 10^{11} \mathrm{erg} \mathrm{cm}^{-2} \mathrm{~s}^{-1}$, shows that the terminal velocity of $\sim 9 \times 10^{7} \mathrm{~cm} \mathrm{~s}^{-1}$, for $r \approx 300 R_{*}$, is changed by only $\sim 0.2 \%$, taking into account the transformation of the radiation energy into the energy of the wind. We conclude, based on this result, that the taking into account of the transformation of the radiation energy into the energy of the wind has only a small affect on the terminal velocity of the wind.

\section{Acknowledgements}

The authors would like to thank the Brazilian agencies FAPESP and CNPq for partial support.

\section{References}

Barlow, M.J., Smith, L.J., Willis, A.J. 1981, MNRAS 196,101

Cassinelli, J.P., van der Hucht, K.A. 1987, in: H. Lamers \& C.W.H. de Loore (eds.), Instabilities in early-type stars (Dordrecht: Reidel), p. 231

Castor, J.I., Abbott, D.C., Klein, R.I. 1975, ApJ 195, 157 (CAK)

dos Santos, L.C., Jatenco-Pereira, V., Opher, R. 1993a, ApJ 410, 732

dos Santos, L.C., Jatenco-Pereira, V., Opher, R. 1993b, $A \& A$ 270, 345

Holzer, T.E., Fla, T., Leer, E. 1983, $A p J$ 275, 808

Jatenco-Pereira, V., Opher, R. 1989a, $A p J$ 344, 513 (JPO)

Jatenco-Pereira, V., Opher, R. 1989b, $A \cup A$ 209, 327 (JPO)

Jatenco-Pereira, V., Opher, R. 1989c, MNRAS 236, 1 (JPO)

Kudritzki, R.P., Hummer D.G. 1990, Ann. Rev. $A \& A$ 28, 303

Lucy, L.B., Solomon, P.M. 1970, ApJ 159, 879

Opher, R., Pereira, V.J.S. 1986, Astrophys. Letters 25, 107 